Article

\title{
Design and Simulation of Electromagnetic Linear Actuators for Jet Dispensers
}

\author{
Minh-Sang Tran and Sheng-Jye Hwang * \\ Department of Mechanical Engineering, National Cheng Kung University (NCKU), Tainan 701, Taiwan; \\ sangtranbk@gmail.com \\ * Correspondence: jimppl@mail.ncku.edu.tw; Tel.: + 886-6-2757575 (ext. 62184)
}

Received: 23 December 2019; Accepted: 24 February 2020; Published: 1 March 2020

\begin{abstract}
Three electromagnetic-based linear actuators, namely a solenoid actuator (SA), a moving coil actuator (MCA), and a moving magnet actuator (MMA), are proposed for driving the needle in a jet dispenser. The total resistance force acting on the needle during operation, including the damping force, the friction force, the inertia force, the compression spring force, and the backpressure, are measured by an experimental model. The thrust force required to overcome this resistance force is then predicted for each actuator using finite element analysis (FEA) simulations. Simple two-dimensional models of the SA, MCA, and MMA are constructed using the same coil dimensions in every case in order to facilitate an objective comparison between them. Simulations in ANSYS Maxwell software are then performed to adjust the specific dimensions of each actuator structure in such a way as to generate the thrust force required to drive the needle in the jet dispenser with the minimum excitation current possible. The simulation results show that for a maximum needle driving frequency of $250 \mathrm{~Hz}$ and a stroke length of $0.5 \mathrm{~mm}$, the excitation current required to generate the necessary thrust force is equal to $1.8 \mathrm{~A}$ and $1.9 \mathrm{~A}$ for the MCA and MMA models, respectively, when a return spring is not used, and 2.2 A, 3.8 A, and 4.1 A for the SA, MCA, and MMA models, respectively, when a return spring is employed. It is additionally shown that the thrust force drop of the MCA and MMA models is far less than that of the SA model, about $0.7 \%, 1.8 \%$, and $61 \%$ for three models, respectively. Three preliminary designs for jet dispensers incorporating the proposed actuators are also generated for reference purposes.
\end{abstract}

Keywords: electromagnetic principle; solenoid actuator; voice coil motor; moving magnet actuator; jet dispenser

\section{Introduction}

Jet dispensers provide an efficient non-contact means of depositing fluid dots or fluid lines with high tolerances on plastic or metal substrates in a very short time. Jet dispensers are thus widely used in fields such as automobile engineering, medical device manufacturing, and electronics assembly [1-8]. The jet dispenser configuration consists of two main parts, namely a jet valve and an actuator. During operation, the needle of the jet valve performs periodic up and down linear motion with a minimum stroke length of approximately $0.3 \mathrm{~mm}$ or above $[9,10]$. As the needle moves with high speed in the downward direction in the dispensing stage, the exceptionally high local pressure produced at the nozzle seat breaks the fluid stream and jets the adhesive out of the nozzle in the form of droplets, which land on the substrate to make dots [11,12].

As shown in Figure 1, the actuator drives the needle through a connecting mechanism. For actuators with a long stroke length, the actuator drives the connecting mechanism directly, as shown in Figure 1a. However, if the shaft stroke of the actuator is less than the minimum stroke of the needle, a magnification mechanism consisting of a fulcrum and a lever arm is required to amplify the actuator stroke, as shown 
in Figure $1 b[9,10,13]$. In this case, the thrust force, $F_{T F}$, generated by the actuator and the resistance force, $F_{R F}$, impacting the needle are related as $F_{T F} * a=F_{R F} * b$, where $\mathrm{a}$ and $\mathrm{b}$ are the lengths of the lever arms on either side of the fulcrum, respectively [14-16].

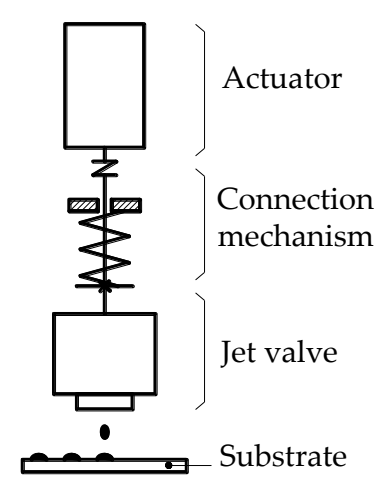

(a)

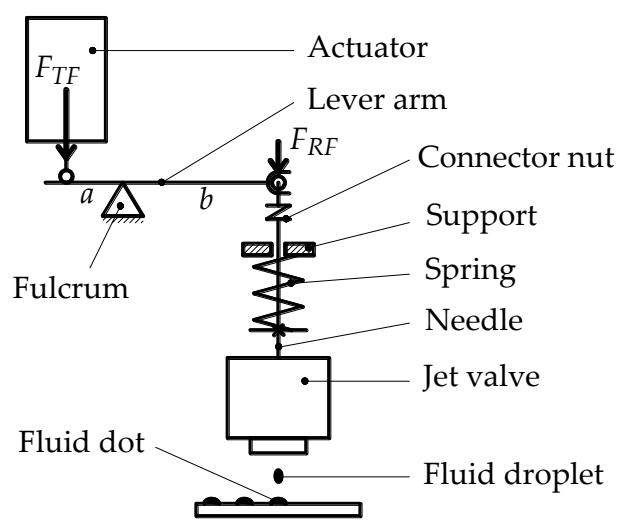

(b)

Figure 1. Jet dispenser models. (a) Direct actuating; and (b) lever arm and fulcrum structure.

Piezo-stack actuators (PSAs) and magnetostrictive actuators (MSAs) generate a high thrust force but have a stroke length of just $100 \mu \mathrm{m}$ [17]. For most practical applications, the stroke length needs to be magnified by a factor of at least three times. Hence, the thrust force, $F_{T F}$, increases accordingly, and energy consumption during the operation increases. Electro-pneumatic actuators (EPAs) and electromagnetic actuators (EMAs) have a smaller thrust force, but longer stroke length, than PSAs or MSAs. The displacement magnification mechanisms are not therefore required for the cases of EPAs and EMAs. The difference between EPAs and EMAs is an electromagnetic actuator used to drive the dispenser needle indirectly or directly. EPAs use a solenoid actuator to actuate open-close states of a pneumatic valve, and this valve controls the pressured air impacting the piston connected the needle to make a linear motion. Meanwhile, the EMA's shaft is connected to the dispenser needle directly, so EMAs drive the needle without intermediary linkages [14]. EMAs have many practical advantages over other types of actuators, including long actuator stroke, low required voltages, simple structure and operation, and low price. Consequently, they are widely used in many different fields nowadays, including computers, digital cameras, packaging and manufacturing, automotive, aerospace, and medicine [18-28].

Current PSA, MSA, and EPA-based jet dispensers can typically reach operating frequencies of 1000 , 250, and $280 \mathrm{~Hz}$, respectively [29-31]. Moreover, EMA-based jet dispensers can also achieve driving frequencies greater than $200 \mathrm{~Hz}$ provided that they have a stroke length of less than $0.5 \mathrm{~mm}$ [32,33]. The linear EMAs have the form of solenoid actuators (SAs), moving coil/voice coil actuators (MCAs), or moving magnet actuators (MMAs). The thrust force versus stroke length characteristics of three types of EMAs is different. When operated at a short stroke length, the thrust force of MCAs and MMAs is approximately constant $[34,35]$, while that of SAs decreases significantly $[36,37]$. Thus, the excitation current applied for SAs must be considerably increased to compensate for the thrust force drop.

The literature contains many studies on the application of EMAs to jet dispensers [14,38]. However, these studies focus only on SAs and MMAs. In other words, the use of MCAs to drive jet dispensers has thus far been ignored. Consequently, the literature lacks a systematic review and comparison of the three actuator structures for jet dispenser applications. Accordingly, the present study designs, enhances, and evaluates three different EMA mechanisms (SA, MCA, and MMA) for driving the jet valve needle in a jet dispenser. The study commences by utilizing an experimental model to measure the total resistance force acting on the jet needle during the dispensing operation. Finite element analysis (FEA) simulations are then performed to determine the thrust force produced by each actuator under typical stroke-length and excitation-current conditions. Further simulations are then performed to 
modify the structure of each actuator in such a way as to generate the thrust force required to overcome the resistance force acting on the needle during dispensing with minimal excitation current possible. Finally, the actuation performance and thrust force magnitude of the three modified actuators are evaluated and compared and preliminary designs for the corresponding actuator-driven jet dispensers prepared. Proposed EMAs-based jet dispensers can be applied to encapsulate light-emitting diode (LED), dispense ultraviolet (UV) cure fluid in electronics assembly, make micro-dots in medicine applications (i.e., blood test, endoscope lens), and encapsulate logos underneath a dome of fluid.

\section{Analysis Method for Electromagnetic Actuators and Design Process}

\subsection{Simplified Electromagnetic Actuators' Model}

The actuators considered in the present study (SA, MCA, and MMA) are all linear electromagnetic actuators (EMAs), which operate based on the force interaction between magnetic fields. The basic configurations of the three actuators are shown in Figure 2a-c, respectively. For all three actuators, the structure comprises two main parts, namely an electric part and a magnetic part. The electric part consists of a current-carrying coil fixed within a coil holder, while the latter part contains a frame/shell and a plunger (SA), fixed magnets (MCA), or movable magnets (MMA). For all three actuators, the movable parts move freely along the z-axis (vertical) direction. When a current is passed through the coil, a magnetic field is produced with poles determined by the direction in which the current flows. The current-induced magnetic field then prompts a motion of the movable parts, as described in the following.

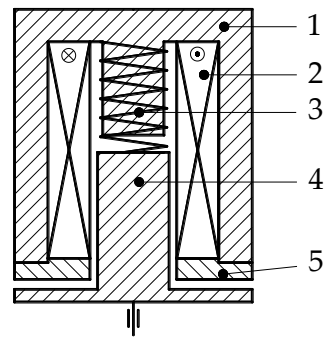

(a)

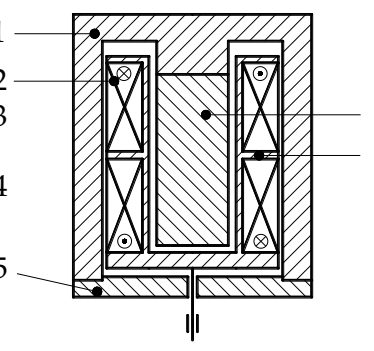

(b)

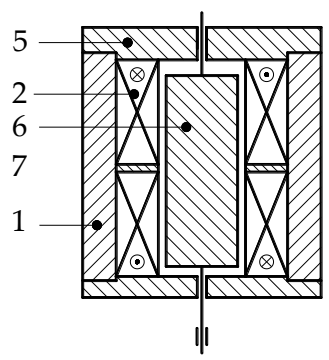

(c)

Figure 2. Basic configurations of electromagnetic actuators. (a) Solenoid actuator (SA); (b) moving coil actuator (MCA), and (c) moving magnet actuator (MMA). 1. Iron shell/frame; 2. coil; 3. spring; 4. plunger; 5 . flange; 6 . magnet; and 7. coil holder.

For the SA, the iron plunger is located within the cylindrical coil and is moved toward the mid-point position of the coil under the effects of the electromagnetic force produced by the actuating current. As the iron plunger moves, it compresses the spring. When the current is subsequently removed, the linear restoring force of the compressed spring pushes the plunger in the opposite direction, producing an actuating effect $[39,40]$.

For the MCA and MMA, the interaction between the permanent magnet and the current-carrying coil generates an electromagnetic force pair that exerts a force of the same magnitude, but in the opposite direction, on the magnetic part and electric part, respectively. The strength of the thrust force depends on both the field strength of the permanent magnet and the magnitude of the excitation current. Moreover, the motion direction of the movable parts depends on the current direction. In other words, both actuators (MCA and MMA) are bi-directional, and may or may not use a return spring to drive the movable parts during the dispensing stage.

As described in the following, the electromagnetic force along the $z$-axis of the SA, MCA, and MMA considered in the present study are investigated by means of finite element analysis (FEA) simulations. 


\subsection{Finite Element Analysis}

In the present study, the magnetic field produced by the electromagnetic actuators is simulated using ANSYS Maxwell software based on the Maxwell equations, which relate the electric and magnetic fields to one another and to the corresponding electric charges and currents. In particular, the simulations use the magnetostatic solution type in Maxwell software, which resolves the static magnetic field existing in a structure that is generated by a direct current (DC) or permanent magnet, as well as the external magnetic field, for both linear and nonlinear materials. Notably, such an approach is applicable to both simple and complex geometries. However, detailed geometries are usually ignored to reduce the possibly disturbing dynamics problem [41,42].

Thus, in the present study, the three-dimensional (3-D) structures of the SA, MCA, and MMA are assumed to be symmetric about the $z$-axis, and are therefore replaced by two-dimensional (2-D) models to simplify the modeling process and reduce the calculation time. ANSYS Maxwell simulations are then performed using the following magnetostatic field equations [42]:

$$
\begin{gathered}
\nabla \times H=J \\
\nabla \cdot B=0 \\
H=\frac{B}{\mu_{r} \mu_{0}} \\
B=\nabla \times A \\
J=\nabla \times\left(\frac{1}{\mu_{r} \mu_{0}} \nabla \times A\right)
\end{gathered}
$$

where $H$ is the magnetic field strength, $J$ is the $z$-component of the current density field in the electric conductor, $B$ is the magnetic flux density, $A$ is the $z$-component of the magnetic vector potential, $\mu_{r}$ is the relative permeability of the material. Note that solving Equations (1)-(5) requires prior knowledge of both the geometric dimensions of the model and the material properties. Furthermore, the relative permeability of nonlinear materials is not constant, and hence the $B$ vs. $H$ curve must also be defined in advance.

\subsection{Design and Analysis Framework}

Figure 3 shows the main steps and procedures in the actuator design and evaluation process performed in the present study. As shown, the framework consists of three parts. In the first part, an experimental model of the jet valve is constructed to measure the total resistance force acting on the needle, $F_{T R F}$, and evaluate the minimum value of the thrust force which each actuator must therefore produce (referred to henceforth as the necessary thrust force, $F_{N T F}$ ). In the second part, numerical models of three simplified 2-D SA, MCA, and MMA models are constructed based on the dimensions proposed. FEA simulations are performed to determine the thrust force, $F_{T F}$, produced by each actuator when excited by a fixed electric current (referred to as initial excitation current, $I$ ). The geometric dimensions of each model are then modified in such a way as to generate a maximum thrust force corresponding to effective SA, MCA and MMA models. In the third part, the minimum excitation current, $I_{j}$, found to satisfy the thrust force, $F_{T F}$, generated by the modified models will be greater than or equal to $F_{N T F}$. Finally, the actuation performance and thrust force magnitude of the three modified actuators are evaluated and compared and preliminary designs for the corresponding actuator-driven jet dispensers also prepared. 


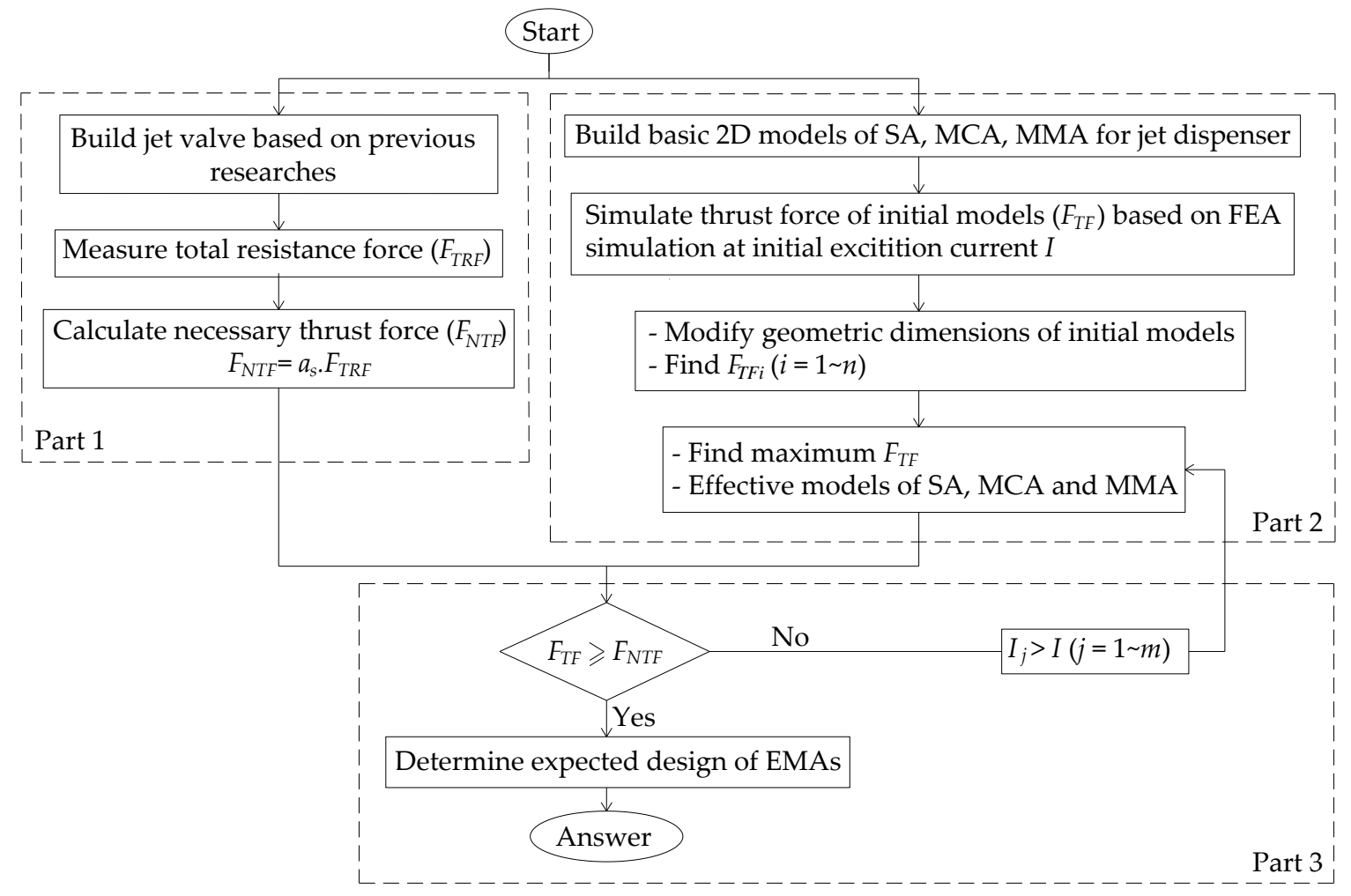

Figure 3. Design and analysis framework for SA, MCA, and MMA mechanisms.

\section{Experimental Measuring of Necessary Thrust Force}

Before designing the SA, MCA, and MMA actuators, an experimental model of a jet valve was constructed to determine the magnitude of the necessary thrust force. In constructing the model, the design dimensions were referenced directly from previous studies in order to reduce the design time $[14,43]$. The jet valve configuration is shown in Figure 4, in which the main geometric dimensions are listed in Table 1 [12]. Note that the other dimensions of the valve that do not affect the flow rate or jetting process of the dispensed liquid (e.g., adhesive) were simply selected in such a way as to facilitate a straightforward assembly of the jet valve parts and the subsequent integration of the jet valve with the actuator and dispenser holder. The nozzle and needle were both fabricated of S304 stainless steel, while the flange, fluid housing and nut were made of Al6061 aluminum alloy. Finally, the jet valve was fitted with commercial Misumi O-rings (NPB No. 3).

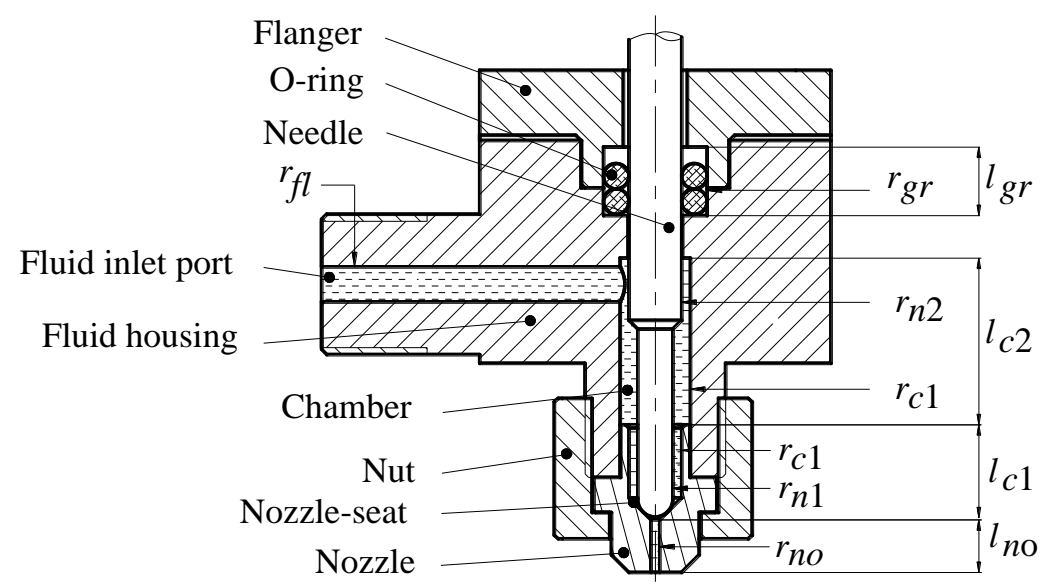

Figure 4. Jet valve configuration. 
Table 1. Main geometric dimensions of experiment jet valve.

\begin{tabular}{cccc}
\hline Geometric Dimension & Symbol, Value (mm) & Geometric Dimension & Symbol, Value (mm) \\
\hline Needle radius & $r_{n 1}=1 ; r_{n 2}=2$ & O-ring groove radius & $r_{o g}=3.9$ \\
Nozzle radius & $r_{n o}=0.25$ & O-ring groove length & $l_{o l}=5.2$ \\
Chamber radius & $r_{c 1}=1.5 ; r_{c 2}=2$ & Chamber length & $l_{c 1}=9.5 ; l_{c 2}=5.4$ \\
Fluid line radius & $r_{f l}=1$ & Nozzle length & $l_{n o}=3$ \\
\hline
\end{tabular}

In the jet valve shown in Figure 4, the adhesive or other dispensed liquid is contained in a reservoir and pressurized by compressed air. During the rising stage of the needle, the pressured adhesive passes the fluid inlet port and enters the dispensing chamber. In the dispensing stage, the needle moves in the downward direction at high speed and collides with the nozzle seat. The resulting high local pressure in the nozzle-seat region causes an amount of the adhesive to be jetted out of the nozzle gate and to fall on the substrate below. The total resistance force acting on the needle as it moves in the downward direction is given by

$$
F_{T R F}=F_{F O}+F_{M}+F_{D}+F_{L}
$$

where $F_{F O}$ is the friction force between the O-rings and the needle, $F_{M}$ is the inertia force, $F_{D}$ is the damping force of the adhesive, and $F_{L}$ is the entire lifting force of the pressured adhesive.

Figure 5 presents a photograph of the experimental setup used to measure $F_{T R F}$. It is noted that the inertia force, $F_{M}$, cannot be identified directly using this experimental model since the needle velocity needs to be calculated separately. Thus, $F_{M}$ was calculated separately and then simply added to the sum of $F_{F O}+F_{D}+F_{L}$ determined experimentally. The experimental setup was built around a ZNHBM-10 kg load sensor positioned in such a way that the sensor head contacted the tail of the jet valve needle. A Mitutoyo micrometer with a range of $0-25 \mathrm{~mm}$ was used to adjust the needle position to obtain a stroke length range of $0-0.5 \mathrm{~mm}$. The dispensing trials were performed using glycerol with a viscosity of $950 \mathrm{cp}$ of Shimakyu Chemical Co., Ltd. Moreover, the compressed air was supplied by a standalone compressor (Model: SVU(P)-203 manufactured by Tong Cheng Iron Works Co., Ltd. (Taichung, Taiwan)) and was passed through a pressure valve (Model: MAR200-8A-2K manufactured by Mindman Industrial Co., Ltd. (Taipei, Taiwan)) in order to maintain constant backpressure on the adhesive surface. An HX711 analog-to-digital converter provided by SparkFun Electronics (Colorado, United States) was used to amplify the signal received from the load sensor and then send it to an Arduino board for processing. Finally, the Arduino board was connected to a laptop to visualize the measured load data. The measurement results showed that the total resistance force acting on the needle (excluding the inertia force, i.e., $F_{F O}+F_{D}+F_{L}$ ) was equal to 3.6, 4.3, 4.8, 5.4, and $6 \mathrm{~N}$ for backpressures of $0,0.5,1,1.5$, and 2 bar, respectively.

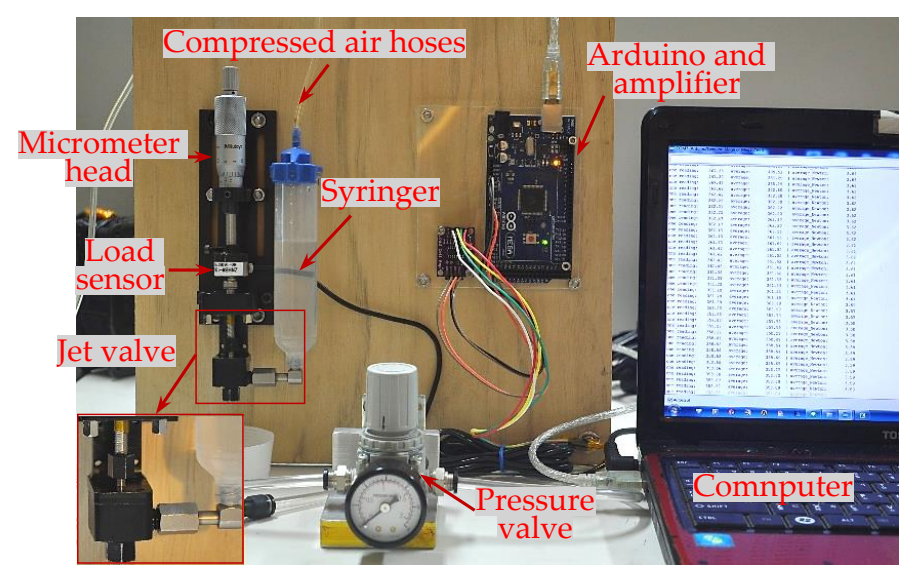

Figure 5. Experimental setup used to measure resistance force acting on jet valve needle during operation. 
The acceleration force, $F_{M}$, was calculated directly as

$$
\begin{gathered}
F_{M}=a \cdot m_{T} \\
m_{T}=m_{n}+m_{m}+m_{s}
\end{gathered}
$$

where $m_{T}, m_{n}, m_{m}$, and $m_{s}$ are the total mass, the needle mass, coil winding/magnet/plunger mass, and the sub-components' mass, respectively; and $a$ is the acceleration. The $m_{T}$ value was estimated to be approximately $0.031 \mathrm{~kg}$. Assume that the jet dispenser operates at a frequency of $250 \mathrm{~Hz}$, and the time required for the needle to complete the full stroke length of $0.5 \mathrm{~mm}$ in the rising or dispensing stage is $1.5 \mu \mathrm{s}$, the needle velocity increases from $0 \mathrm{~m} / \mathrm{s}$ to the maximum velocity at the end of the stroke length, and the average velocity and acceleration are calculated based on equations, $v=\Delta x / \Delta t$ and $a=\Delta v / \Delta t$, then calculated average acceleration is equal to approximately $220 \mathrm{~m} / \mathrm{s}^{2}$. The inertia force, $F_{M}$, is then determined from Equation (7) to be $6.8 \mathrm{~N}$ at the maximum backpressure of $0.5 \mathrm{bar}$.

In practice, the magnitude of the necessary thrust force $\left(F_{N T F}\right)$ to be produced by the actuators depends on whether or not a return spring is used to assist the needle motion in the dispensing stage. For the SA, the thrust force acts only in one direction, and hence a return spring must be used. However, for both the MCA and the MMA models, the thrust force may act in either direction (up or down) and hence a return spring can be employed if desired. Thus, the following formulations for $F_{N T F}$ are derived

$$
\begin{gathered}
F_{N T F}=a_{S} \cdot F_{T R F} \\
F_{N T F S}=a_{S} \cdot\left(F_{T R F}+F_{S}\right)
\end{gathered}
$$

where $F_{N T F}$ and $F_{N T F S}$ denote the necessary thrust force without and with a spring, respectively. In addition, as is a safety factor (described in the following paragraph) and $F_{S}$ is the spring force.

From Equation (6), the total resistance force, $F_{T R F}$, is equal to $11.1 \mathrm{~N}$ for backpressure of 0.5 bar. However, to ensure that the thrust force produced by the actuator under realistic working conditions characterized by a temperature change in the coil windings or variations in the adhesive viscosity, for example, the total resistance force should be multiplied by a safety factor, $a_{s}$, as shown in Figure 3 . Assuming that $a_{S}$ is assigned a value of 1.2, the value of $F_{N T F}$ is determined to be $13.3 \mathrm{~N}$ (Equation (9)) if no return spring is used. For the case, where a spring is used to drive the needle in the dispensing stage, the spring force value, $F_{S}$, can be set equal to the same $F_{N T F}$ value. In other words, the necessary thrust force, $F_{N T F S}$, is determined from Equation $(10)$ to be $29.3 \mathrm{~N}$ (i.e., $1.2 \times(11.1+13.3) \approx 29.3$ ).

\section{Proposal Actuators' Model}

As described in Section 2, the aim of the second part of the design framework was to modify the three proposal actuator designs in such a way as to generate the biggest thrust force. To simplify the modeling process, and reduce the computation time, the actuator models were further simplified to the forms shown in Figure 6. As shown, each model was constructed using only the most essential features of the actuator structure. For example, the SA model contains only the plunger, the iron core, and the coil. Similarly, the MCA and MMA models comprise only the permanent magnet, two iron-rings, two serial coils, the shell, and an insulated aluminum ring. Note that for all three actuators, the remaining components were simply ignored since they are either made of non-magnetic materials such as aluminum and rubber, or have no effect on the magnitude of the simulated thrust force.

The modeling process comprised two steps. In the first step, the 2-D axisymmetric models of the SA, MCA, and MMA were constructed using the same basic dimensions of the coil, including the coil height and the inner and outer diameters. Note that such an approach was deliberately taken in order to facilitate an objective comparison between the actuation performance of the three models. In the second step, specific geometric variables were added to each basic model, depending on the particular actuator involved, and assigned appropriate design values. For example, variable $d$, was added to the SA model (see Figure 6a), while variable $e$ was added to the MCA and MMA models (see Figure 6b,c). 
Note that the MCA and MMA models are the same and differ only in whether the coil windings (MCA) or magnets (MMA) move during the dispensing process. Table 2 summarizes the specific geometric dimensions of three basic models prior to their modification.

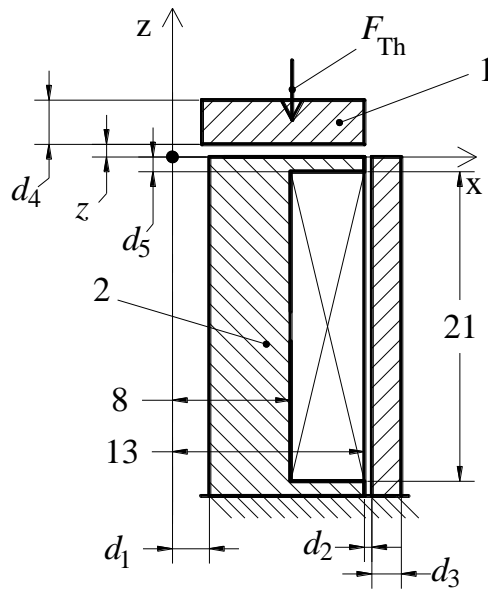

(a) SA

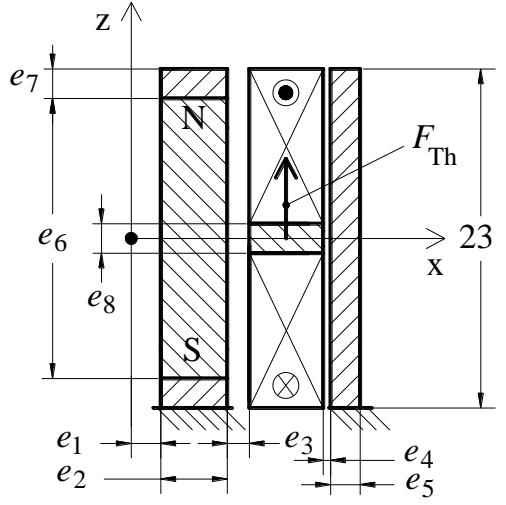

(b) MCA

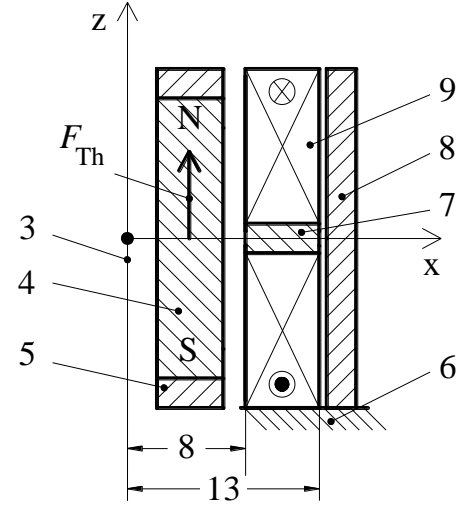

(c) MMA

Figure 6. 2-D axisymmetric models of electromagnetic actuators. (a) Solenoid actuator (SA); (b) moving coil actuator (MCA); and (c) moving magnet actuator (MMA). 1. Plunger; 2. iron core; 3. axis of symmetry; 4 . magnet; 5 . iron-ring; 6 . fixed support; 7 . insulated aluminum ring; 8. shell; and 9. coil.

Table 2. Basic geometric dimensions of models prior to modification.

\begin{tabular}{cccccccccc}
\hline Symbol & $\begin{array}{c}\text { Value } \\
(\mathbf{m m})\end{array}$ & Symbol & $\begin{array}{c}\text { Value } \\
\mathbf{( m m )}\end{array}$ & Symbol & $\begin{array}{c}\text { Value } \\
(\mathbf{m m})\end{array}$ & Symbol & $\begin{array}{c}\text { Value } \\
(\mathbf{m m})\end{array}$ & Symbol & $\begin{array}{c}\text { Value } \\
(\mathbf{m m})\end{array}$ \\
\hline$d_{1}$ & 2.5 & $d_{4}$ & 2 & $e_{1}$ & 2 & $e_{4}$ & 0.5 & $e_{7}$ & 0 \\
$d_{2}$ & 0.5 & $d_{5}$ & 1 & $e_{2}$ & 4.5 & $e_{5}$ & 2 & $e_{8}$ & 2 \\
$d_{3}$ & 2 & - & - & $e_{3}$ & 1.5 & $e_{6}$ & 23 & - & - \\
\hline
\end{tabular}

In constructing the actuator models, the coil windings were assumed to be fabricated of copper with a relative permeability of $\mu_{r}=1$, while the magnets in the MCA and MMA models were assumed to be fabricated of NdFeB with a relative permeability of $\mu_{r}=1.05$ and a residual magnetism of $B_{r}=1.43 \mathrm{~T}$. The core and shell of the SA model, together with the iron rings and shell of the MCA and MMA models, were assumed to be fabricated of AISI 1020 carbon steel. AISI 1020 is a nonlinear material, and hence the relative permeability, $\mu_{r}$, has a non-constant value. Accordingly, the $B-H$ curve was defined in the form shown in Figure 7 [44]. Finally, the insulated ring in the MCA and MMA models was fabricated of Al6061 aluminum alloy with a relative permeability of $\mu \mathrm{r}=1$. Figure 8 shows the boundary conditions imposed on each of the actuator models.

The goal of the simulation process was to determine the force acting on the movable parts of the actuators, namely the plunger in the SA model, the coil windings in the MCA model, and the magnet core in the MMA model. For the SA model, the actuator was assumed to comprise a single-coil winding with 900 turns. By contrast, the MCA and MMA models were assumed to have multiple coil windings with 450 turns on each winding. For all of the models, the DC current in the cross-section of the coils was assumed to be uniformly distributed, with the flow direction of the current perpendicular to the cross-section surface [42]. For the SA model, the initial $z$ gap was set as $0.7 \mathrm{~mm}$ and the plunger then moved toward the core over a stroke length of $0.5 \mathrm{~mm}$. For the MCA and MMA models, the mid-points of the magnets and coil windings were set to coincide initially and the magnets then moved through a distance of $0.5 \mathrm{~mm}$ away from this mid-point position in a direction governed by the direction of the current flow through the coil. 


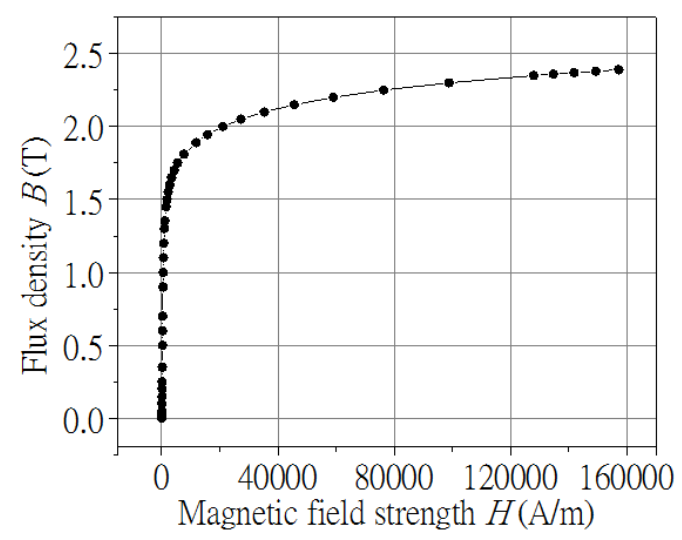

Figure 7. B-H curve of AISI 1020 carbon steel.

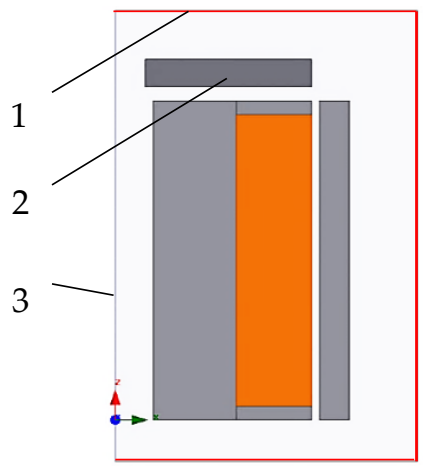

(a) SA model

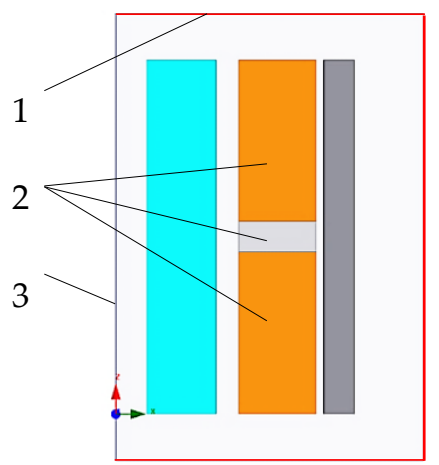

(b) MCA model

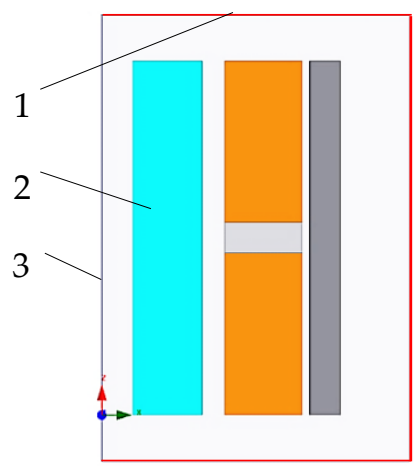

(c) MMA model

Figure 8. Boundary conditions imposed on 2-D symmetric models. (a) Balloon boundary; (b) movable part; and (c) symmetric axis.

As described in the following section, the thrust force produced by each actuator was calculated for various values of the specific design variables $(d$ or $e$ ) in order to determine the geometry design which enhanced the thrust force. In this article, the models were modified according to whole-scale numerical experiment results. The input parameters are $d_{1} \sim d_{4}$ for SA and $e_{1} \sim e_{8}$ for MCA and MMA, and the objective parameter is the thrust force, $F_{T F}$. Input variables are assigned the lower and upper bounds. When solving the problem, each input variable changed its value in turn, while the others were fixed to find the higher value of thrust force. The simulation results were then used to determine the minimum value of the applied current sufficient to generate a thrust force greater than the necessary thrust force. Finally, an investigation was performed into the smaller magnitude variation of the thrust force as the actuators were displaced through a total stroke length of $0.5 \mathrm{~mm}$.

\section{Results and Discussion}

\subsection{Models, Convergence, and Thrust Force after Modification}

As described in the previous section, two specific geometry variables ( $d$ and $e$ ) were assigned to the SA and MCA/MMA actuators, respectively, and simulations were then performed to determine the geometry settings which maximized the actuator thrust with the minimal current. Table 3 shows the considered design range for each variable and the value found to maximize the actuator thrust force. Figure 9 shows the models of the SA, MCA, and MMA before and after the modification process, respectively. 
Table 3. Variable range of geometric dimensions and modified dimensions.

\begin{tabular}{cccccc}
\hline Symbol & $\begin{array}{c}\text { Variable } \\
\text { Range }\end{array}$ & $\begin{array}{c}\text { After } \\
\text { Modification }\end{array}$ & Symbol & $\begin{array}{c}\text { Variable } \\
\text { Range }\end{array}$ & $\begin{array}{c}\text { After } \\
\text { Modification }\end{array}$ \\
\hline$d_{1}$ & $2.5-4$ & 2.5 & $e_{3}$ & $1-2$ & 1.25 \\
$d_{2}$ & $0.3-1$ & 0.4 & $e_{4}$ & $0.3-1$ & 0.6 \\
$d_{3}$ & $1-2.5$ & 1.7 & $e_{5}$ & $1-2.5$ & 1.5 \\
$d_{4}$ & $1-3$ & 3 & $e_{6}$ & $5-23$ & 13 \\
$d_{5}$ & $0.5-1.5$ & 0.8 & $e_{7}$ & $0-4$ & 1 \\
$e_{1}$ & $2-4$ & 2 & $e_{8}$ & $0.5-3$ & 1.6 \\
$e_{2}$ & $3-5$ & 4.75 & - & & \\
\hline
\end{tabular}

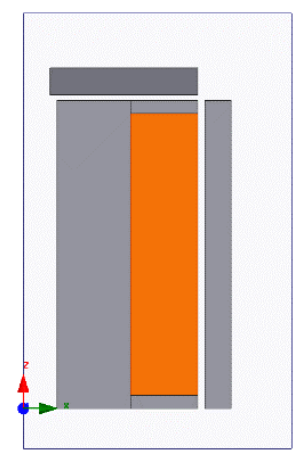

(a)

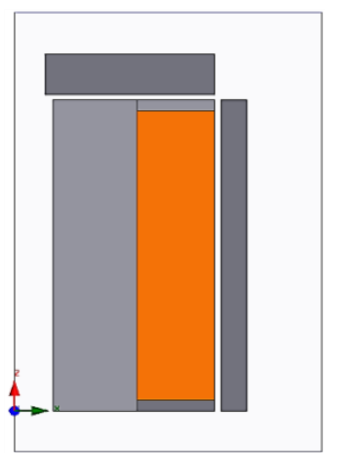

(b)

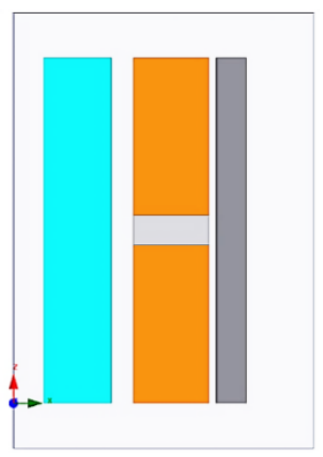

(c)

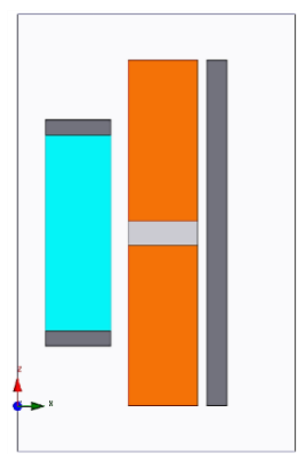

(d)

Figure 9. Models before and after modification. $(\mathbf{a}, \mathbf{b})$ SA model before and after modification; and (c,d) MCA and MMA models before and after modification.

Figure 10 shows the convergence plot of the thrust force by varying the mesh density in each case given an excitation current of $2 \mathrm{~A}$ at the initial position of $z(0.2 \mathrm{~mm}$ for SA and $0 \mathrm{~mm}$ for both MCA and MMA). The thrust forces generated by SA, MCA, and MMA models start to reach a good convergence when mesh element numbers are higher than 5000, 8000, 8000 elements, respectively. Accordingly, the thrust forces approximately converge at $63.42,15.65$, and $14.72 \mathrm{~N}$ for SA, MCA, and MMA, respectively. Although a higher mesh density enhances the accuracy of predicted thrust forces, it significantly increases computation time. Consequently, the number of mesh elements mentioned above for each model is reliable enough and is used for setting mesh operations in the next simulation steps.

Figure 11 shows the meshed geometric models and magnetic field distribution at $I=2 \mathrm{~A}$ of SA, MCA and MMA after modification, respectively. For SA model, the biggest values of the magnetic field at the position of corners on the coil holder exceeds saturation value because of the edge and thin wall effects. As described above, the MCA and MMA structures are essentially similar, with the only difference between them being whether the coil windings (MCA) or magnet (MMA) move during operation. As a result, the two actuators have similar magnetic flux distribution. The highest value of the magnetic field concentrates on iron-rings where are close to the coil.

Figure 12 shows the simulated thrust force for the three models before and after the modification process. It is seen that for every model, the re-design process increases the thrust force produced by the actuator. From inspection, the average percentage improvements in the thrust force of the SA, MCA, and MMA models are found to be about $27.5 \%, 36 \%$, and $48 \%$, respectively, over the considered actuation current range of 0.6-2.6 A with increments of $0.2 \mathrm{~A}$. 


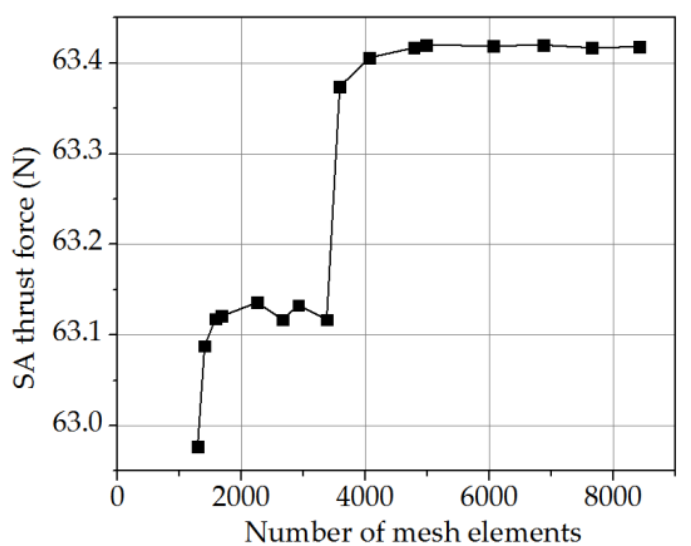

(a) SA model.

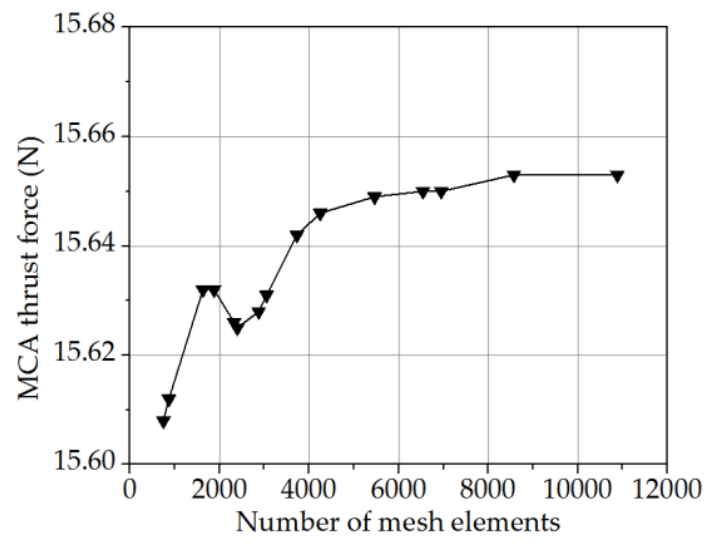

(b) MCA model

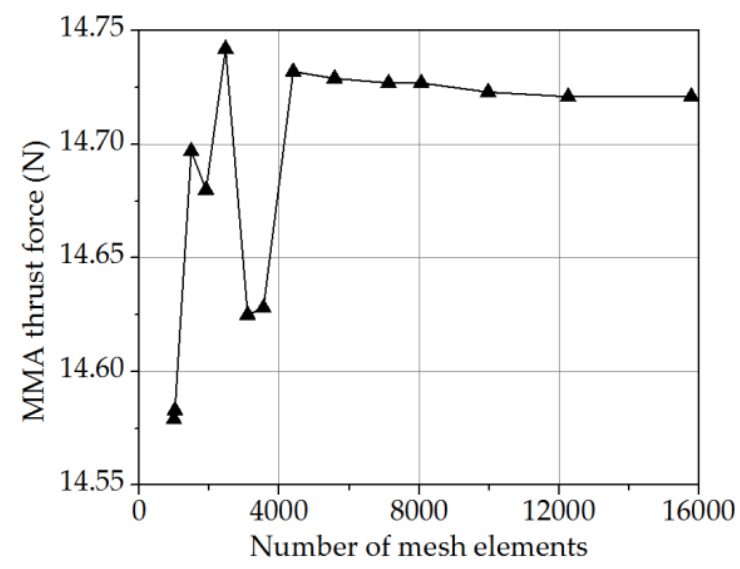

(c) MMA model

Figure 10. Thrust force convergence of modified models.
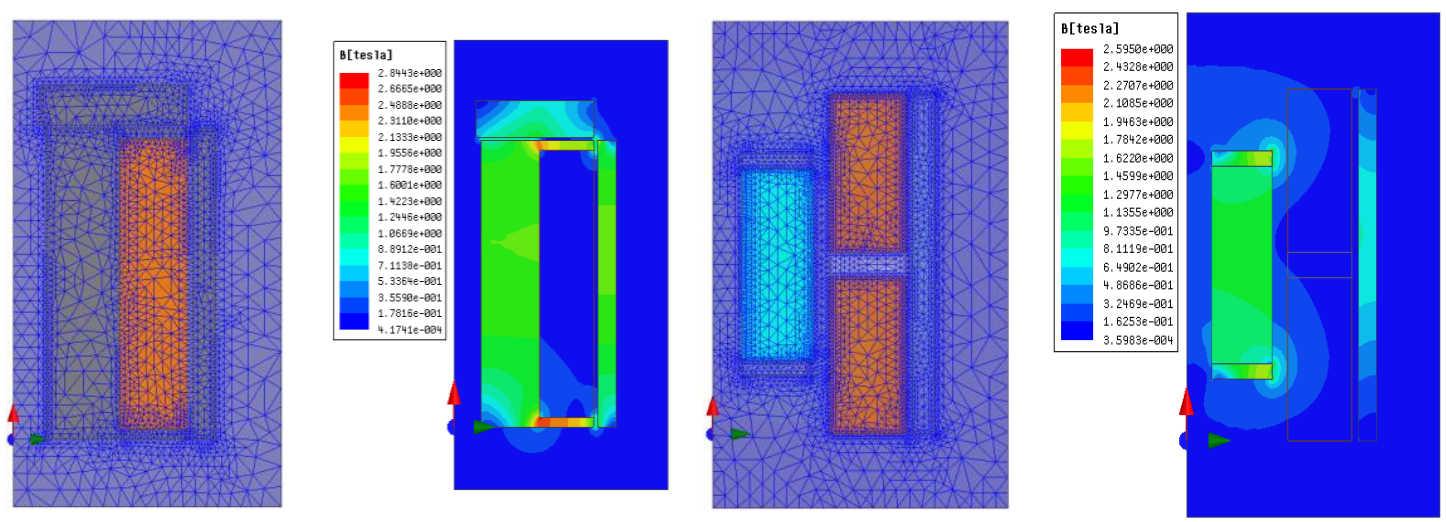

(a)

(b)

Figure 11. Meshed geometry and magnetic field distribution of models after modification. (a) SA model with 5118 mesh elements, and (b) MCA and MMA models with 8612 mesh elements. 


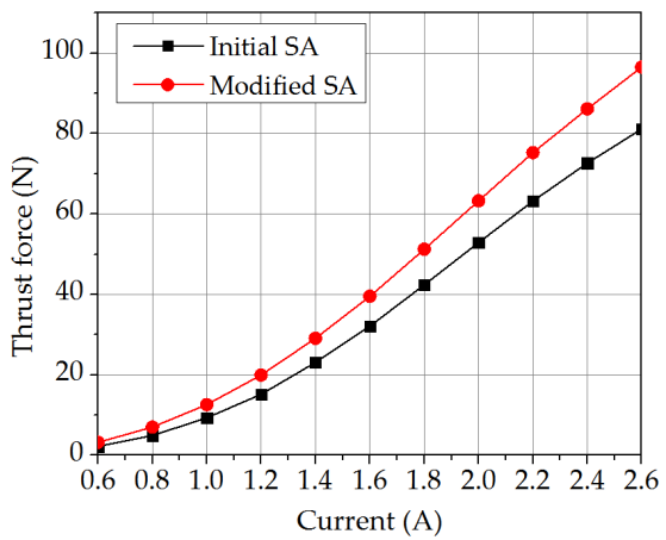

(a) SA thrust force at $z=0.2$

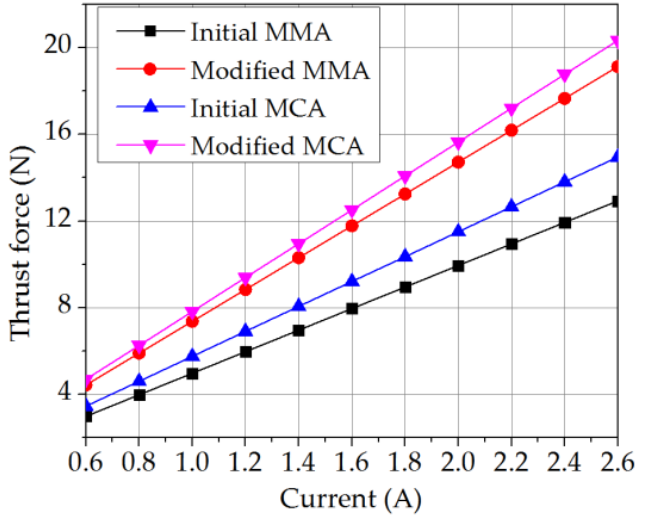

(b) MCA and MMA at $z=0$

Figure 12. Thrust force of three models before and after modification.

\subsection{The relationship Among Applied Current, Stroke Length, and Thrust Force}

The modified SA, MCA, and MMA models were used to further investigate the effects of the stroke length and excitation current on the thrust force value. In performing the simulations, the $z$ gap of the SA model was increased from $0.2-0.7 \mathrm{~mm}$, while the coil windings of the MCA model and magnet core of the MMA model were displaced through a $z$ gap of $0.0-0.5 \mathrm{~mm}$. For each actuator, the thrust force was calculated at intervals of $0.5 \mathrm{~mm}$ along the stroke length. In addition, the simulations were performed at intervals of every $0.2 \mathrm{~A}$ in the considered current range (1-2 A for the SA and 1.6-2.6 A for the MCA and MMA). Figure 13 shows the corresponding simulation results. For each actuator, the thrust force increases approximately linearly with increasing current. Furthermore, for each model, the magnitude of the thrust force varies with the position of the movable part (i.e., plunger, coil, or magnet). For the SA model, the thrust force decreases by around $61 \%$ on average as the $z$ gap increases from 0.2-0.7 mm. For the MCA and MMA models, the thrust force attains its highest value at the center position $(z=0 \mathrm{~mm})$. However, as the coil or magnet moves away from this position, the thrust force drops by around $0.7 \%$ for the MCA model and $1.8 \%$ for the MMA model as $z$ increases to $0.5 \mathrm{~mm}$. Overall, the SA thrust force decreases significantly when the position of the plunger is far from the coil holder. For the MCA and MMA models, the thrust force appears to remain constant as the stroke length increases. However, a detailed inspection of the simulation results shows that $F_{M C A}$ and $F_{M M A}$ actually drop within a narrow range of around $0.2 \mathrm{~N}$ and $0.3 \mathrm{~N}$, respectively.

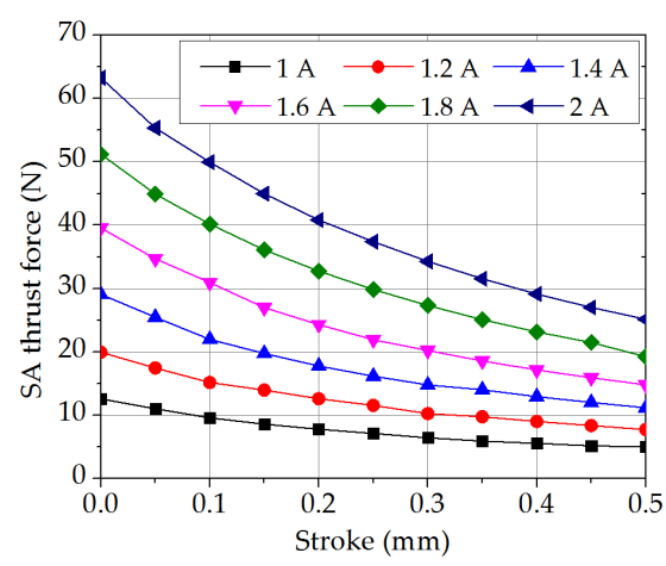

(a) SA thrust force

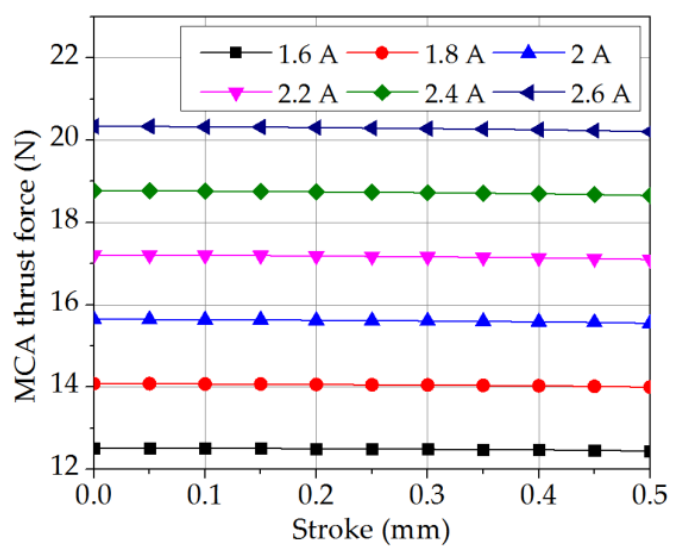

(b) MCA thrust force

Figure 13. Cont. 


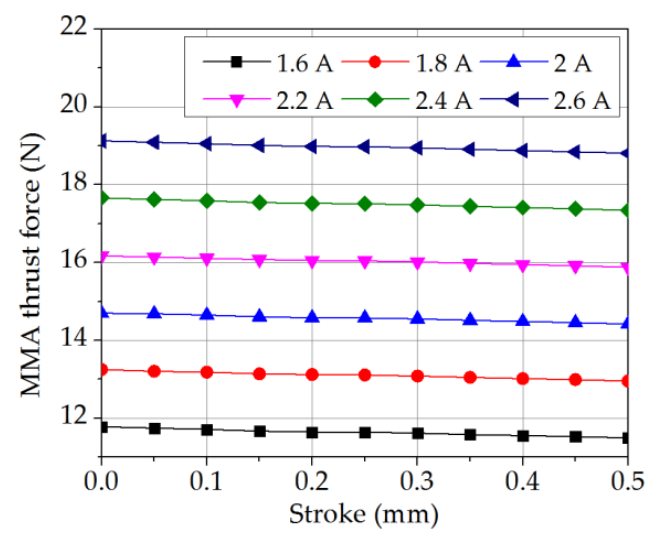

(c) MMA thrust force

Figure 13. Thrust force, applied current, and stroke length relationships.

\subsection{Minimum Excitation Current}

As described above, the thrust force produced by the actuators varies in accordance with the magnitude of the excitation current and the position of the moveable parts. For practical implementations, the stroke length is fixed (e.g., $0.5 \mathrm{~mm}$ ), and the thrust force is therefore dominated by the excitation current. The experimental results presented in Section 3 have shown that for the jet dispenser considered in the present study, the thrust force generated by the actuators must be at least 13.3 $\mathrm{N}$ when a return spring is not used and $29.3 \mathrm{~N}$ when a spring is used. For the SA, the thrust force, $F_{S A}$, can act in only one direction. Hence, a return spring must be used to return the plunger to its original position after each dispensing operation. However, for the MCA and MMA models, the thrust force $\left(F_{M C A}\right.$ and $\left.F_{M M A}\right)$ can work in either direction and hence a return spring may or may not be fitted. Figure 14 shows the thrust force produced by the MCA and MMA models over the stroke-length range of $0.5 \mathrm{~mm}$ for two values of the excitation current when a spring is not fitted. As shown, the values of $F_{M C A}$ and $F_{M M A}$ obtained for currents of $1.7 \mathrm{~A}$ and $1.8 \mathrm{~A}$, respectively, are lower than the required thrust force over the majority of the stroke length. By contrast, $F_{M C A}$ and $F_{M M A}$ exceed $13.3 \mathrm{~N}$ for all values of $z$ given excitation currents of $1.8 \mathrm{~A}$ and $1.9 \mathrm{~A}$. Hence, the minimum values of the excitation current required to actuate the jet dispenser with no spring are determined to be $I_{\min M C A}=1.8 \mathrm{~A}$ and $I_{\text {minMMA }}=1.9 \mathrm{~A}$, respectively. Figure 15 shows the magnitude of the thrust force with the stroke length for the case where a return spring is fitted to the actuation mechanism. For the SA model (Figure 15a), the thrust force produced using an excitation current of $2.1 \mathrm{~A}$ exceeds the required thrust force for most of the stroke length. However, for values of $z$ greater than $0.45 \mathrm{~mm}$, the thrust force falls below the required value. Thus, the minimum value of the excitation current is set as $I_{\min S A}=2.2 \mathrm{~A}$. Similarly, from Figure $15 \mathrm{~b}, I_{\min M C A}$ and $I_{\min M M A}$ are set as $3.8 \mathrm{~A}$ and $4.1 \mathrm{~A}$, respectively.

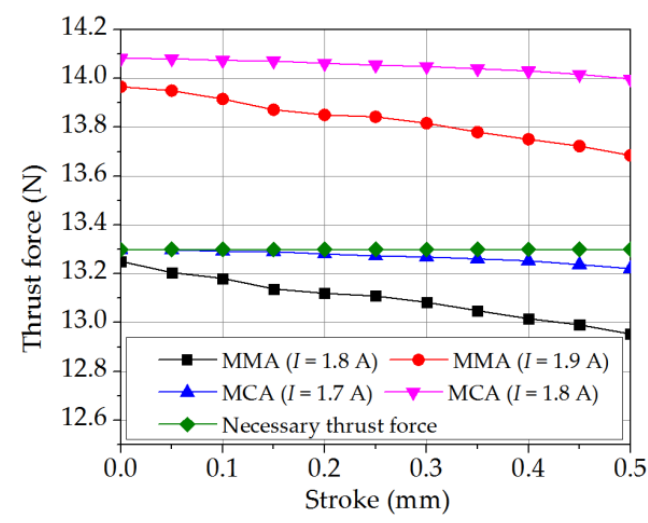

Figure 14. Minimum excitation current for MCA and MMA models (no return spring). 


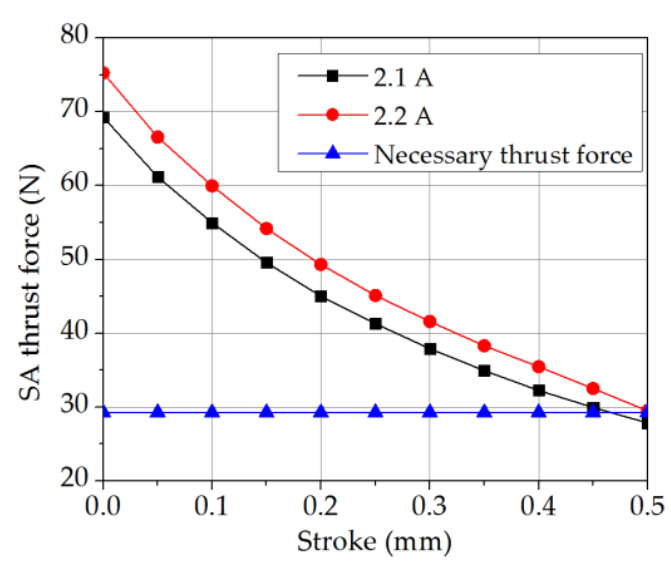

(a) For SA

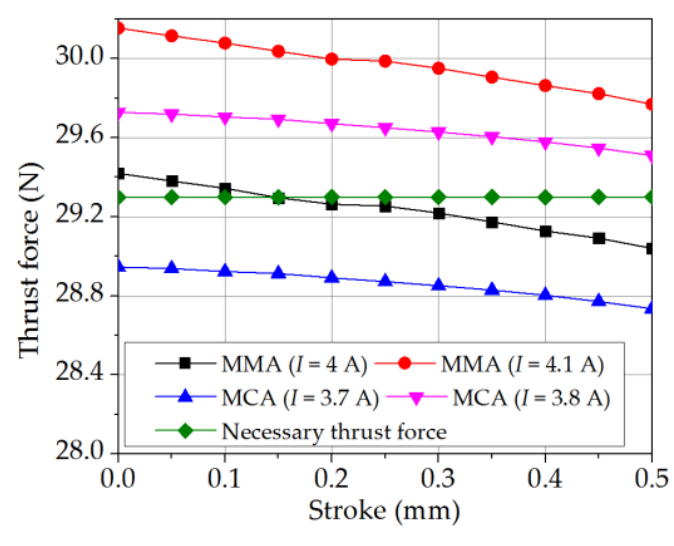

(b) For MCA and MMA

Figure 15. Minimum excitation current for (a) SA, (b) MCA, and MMA models (with return spring).

\subsection{Proposed Jet Dispenser Designs}

The present results show that the SA model operates well over a short stroke length and develops a high thrust force with a minimum excitation current of 2.2 A. SA is best suited for the jet dispenser applications when using a return spring. However, for a longer stroke length, the excitation current must be significantly increased in order to compensate for the big drop in $F_{S A}$. For the MCA and MMA models considered in the case of no spring, the MCA model provides a more suitable solution since it has a low thrust force drop (i.e., $0.7 \%$ ) and a low excitation current (i.e., $1.8 \mathrm{~A}$ ) over a long stroke range.

The results presented in the preceding sections have also confirmed that, given an appropriate design, the SA, MCA and MMA all provide a sufficient thrust force to drive the needle of the considered jet dispenser. In addition, for all three mechanisms, the magnitude of the generated thrust force varies with the relative position between the core and the movable parts of the actuator. For example, for the SA, the thrust force reduces dramatically as the plunger moves further away from the core. Furthermore, since the attraction force between the plunger and the core is strong only when the plunger is close to the core, then irrespective of the current direction in the coil, a return spring is required to push the needle back during the dispensing stage. In the MCA and MMA designs, the largest thrust force is produced when the magnet lies entirely inside the coil windings and the mid-points of the magnets and coils overlap. When the direction of the current flowing through the coil reverses, the direction of the interaction force between the magnet and the coil also reverses. Hence, both actuator mechanisms are capable of producing bi-directional motion and can therefore drive the needle in both the rising stage and the dispensing stage without the need for a return spring. However, a return spring can be incorporated into the mechanism if desired to further actuate the needle in the dispensing stage; thus ensuring that the nozzle gate is fully closed by the needle tip at the end of the dispensing operation such that no leakage occurs. However, this performance advantage is obtained at the expense of a larger thrust force, $F_{\text {NTFS }}$ (i.e., more than twice that required when no return spring is employed).

Figure 16 shows the basic configurations and dimensions of the three EMA-based jet dispensers developed in the present study. Note that the actuators are based on the modified design variables shown in Table 3, while the dimensions of the other parts, e.g., the coil holder, the flange, the stroke bolt, and the housing, are based on experience. Moreover, the primary dimensions of the jet valve are indicated in Table 1 and the sub-dimensions of the jet valve components are chosen in such a way as to facilitate the assembly process and ensure proper dispensing operation. 


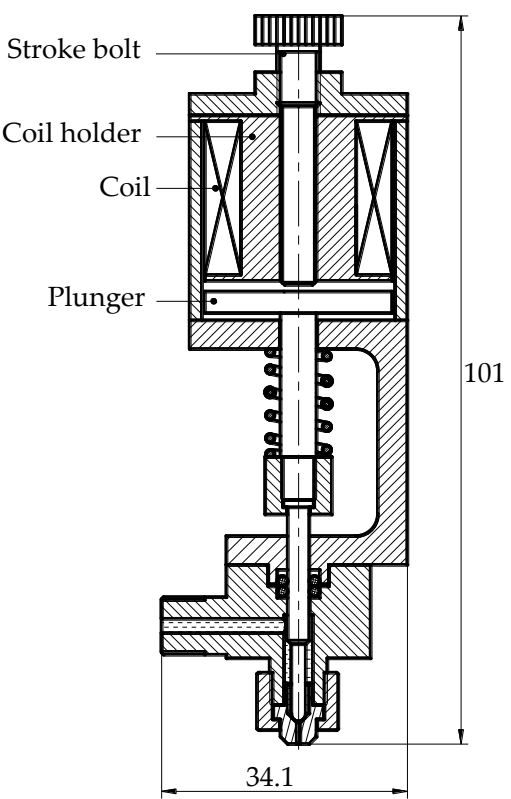

(a) SA jet dispenser

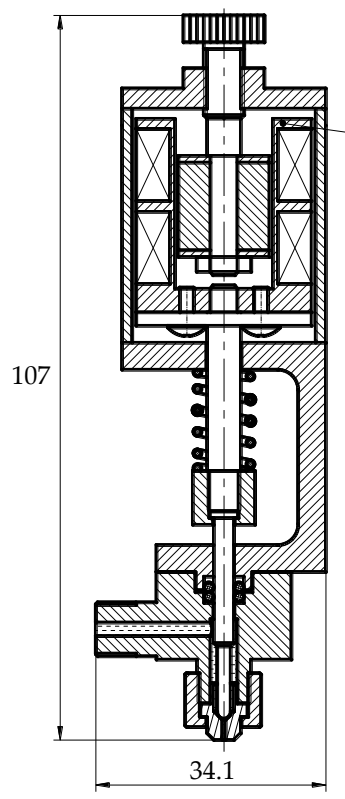

(b) MCA jet dispenser

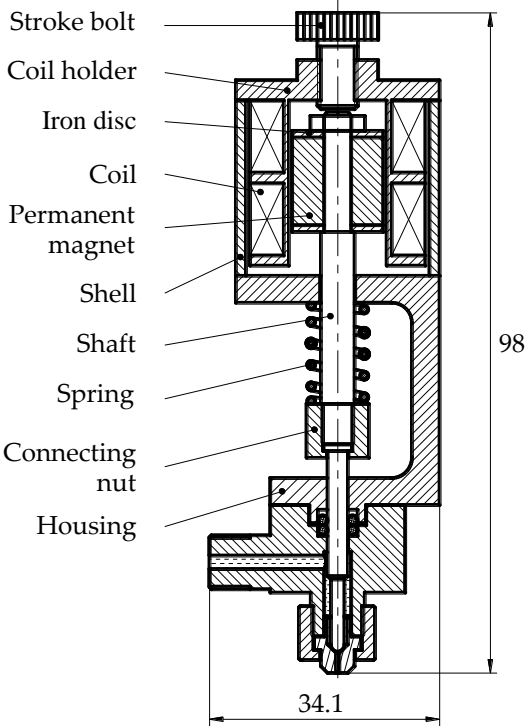

(c) MMA jet dispenser

Figure 16. Dispenser configuration of jet dispensers based on proposed actuators.

\section{Conclusions}

This study has utilized the finite element method (FEM) simulation approach to design, modify and characterize the performance of three electromagnetic actuators (namely a solenoid actuator (SA), a moving coil actuator (MCA) and a moving magnet actuator (MMA)) for jet dispensing applications. The total actuation force required to drive the needle in the jet dispenser has been determined using an experimental model. FEM simulations have been performed to modify the geometries of the three proposal actuators in such a way as to achieve the thrust force required to actuate the needle in the jet dispenser with the minimum excitation current possible. The average percentage improvements of the thrust force for the SA, MCA, and MMA after modification are about $27.5 \%, 36 \%$, and $48 \%$, respectively. Comparing the three actuator mechanisms, the SA generates a high thrust force in the initial position. However, the thrust force drops by approximately $61 \%$ when the plunger moves through a stroke length of $0.5 \mathrm{~mm}$. The thrust force drop produced by the MCA and MMA models is far lower; with drops of just $0.7 \%$ and $1.8 \%$, respectively, as the moving parts (i.e., the coil and magnet) move through the same stroke distance of $0.5 \mathrm{~mm}$.

In the absence of a return spring, the excitation current required to achieve the necessary thrust force is equal to $1.8 \mathrm{~A}$ and 1.9 $\mathrm{A}$ for the MCA and MMA models, respectively. When a return spring is optionally fitted to the actuator mechanism, the minimum excitation current increases to $2.2 \mathrm{~A}, 3.8 \mathrm{~A}$, and $4.1 \mathrm{~A}$ for the SA, MCA, and MMA models, respectively. In the two cases without and with a return spring, MCA and SA are the best choices for jet dispenser applications, respectively. Based on the modified simulation models, three preliminary designs for jet dispenser structures integrated EMAs have been proposed for reference purposes. In future research, an experimental model consisting of both the proposed actuators and the EMAs-based jet dispenser will be fabricated in order to verify the accuracy of the simulation results and serve as the basis for further modifications and improvements.

Author Contributions: Methodology, M.-S.T. and S.-J.H.; investigation, M.-S.T.; formal analysis, M.-S.T.; validation, S.-J.H.; writing-original draft preparation, M.-S.T.; writing-review and editing, M.-S.T. and S.-J.H.; and supervision, S.-J.H. All authors have read and agreed to the published version of the manuscript.

Funding: This research received no extend funding.

Conflicts of Interest: The authors declare no conflict of interest. 


\section{References}

1. Chen, Y.; Wang, F.; Li, H.X. Experimental and modeling study of breakup behavior in silicone jet dispensing for light-emitting diode packaging. IEEE Trans. Compon. Packag. Manuf. 2015, 5, 1019-1026. [CrossRef]

2. Gu, S.; Jiao, X.; Liu, J.; Yang, Z.; Jiang, H.; Lv, Q. Design and experiment of a solder paste jetting system driven by a piezoelectric stack. Micromachines-Basel. 2016, 7, 112. [CrossRef] [PubMed]

3. Scoutaris, N.; Nion, A.; Hurt, A.; Douroumis, D. Jet dispensing as a high throughput method for rapid screening and manufacturing of cocrystals. Cryst. Eng. Comm. 2016, 18, 5079-5082. [CrossRef]

4. Babiarz, A.J. Jetting small dots of high viscosity fluids for packaging applications. Semicond. Int. 2006, 29, 2-6.

5. Anthony, F. Advantages of Non-Contact Dispensing in SMT Assembly Processes. In Proceedings of the SMTA International Conference, San Jose, CA, USA, 12-16 September 1999.

6. Li, M.; Tian, X.; Chen, X. A brief review of dispensing-based rapid prototyping techniques in tissue scaffold fabrication: Role of modeling on scaffold properties prediction. Biofabrication 2009, 1, 032001. [CrossRef]

7. Nguyen, Q.-H.; Choi, S.-B.; Kim, J.-D. The design and control of a jetting dispenser for semiconductor electronic packaging driven by a piezostack and a flexible beam. Smart Mater. Struct. 2008, 17, 065028. [CrossRef]

8. Deng, G.; Wang, N.; Zhou, C.; Li, J. A simplified analysis method for the piezo jet dispenser with a diamond amplifier. Sensors 2018, 18, 2115. [CrossRef]

9. Wang, L.; Du, J.; Luo, Z.; Du, X.; Li, Y.; Liu, J.; Sun, D. Design and experiment of a jetting dispenser driven by piezostack actuator. IEEE Trans. Compon. Packag. Manuf. 2012, 3, 147-156. [CrossRef]

10. Jeon, J.; Hong, S.M.; Choi, M.; Choi, S.B. Design and performance evaluation of a new jetting dispenser system using two piezostack actuators. Smart Mater. Struct. 2014, 24, 015020. [CrossRef]

11. Nordson_Corporation. New Solder Paste Jetting System from Nordson EFD Provides Fast, Repeatable Non-Contact Dispensing. Available online: https://www.nordson.com/en/divisions/efd/about-us/news (accessed on 5 August 2019).

12. Essemtec-USA-Ltd. SMT Applications Realized on Our Machines. Available online: https://essemtec.com/ en/applications/ (accessed on 5 August 2019).

13. Choi, S.B.; Han, Y.M. Piezoelectric Actuators: Control Applications of Smart Materials; CRC Press: Boca Raton, FL, USA, 2016; pp. 191-213.

14. Tran, M.S.; Hwang, S.J. Design and Experiment of a Moving Magnet Actuator Based Jetting Dispenser. Appl. Sci. Basel. 2019, 9, 2911. [CrossRef]

15. Ahmadi, M.; Babiarz, A.; Ciardella, R.; Fang, L.; Fiske, E.; Quinones, H.; Ratledge, T. Jetting Dispenser with Multiple Jetting Nozzle Outlets. U.S. Patent 20070145164, 28 June 2007.

16. Clark, J.A.; Fort, W.C.; Gould, M.A.; Ridge, W.M.; Saidman, L.B.; Varga, L.J. Jetting Dispenser and Method of Jetting Highly Cohesive Adhesives. U.S. Patent 8753713, 17 June 2014.

17. John, S.; Sirohi, J.; Wang, G.; Wereley, N.M. Comparison of piezoelectric, magnetostrictive, and electrostrictive hybrid hydraulic actuators. J. Intel. Mater. Syst. Struct. 2007, 18, 1035-1048. [CrossRef]

18. Miu, D.K.; Bhat, S.P. Minimum power and minimum jerk position control and its applications in computer disk drives. IEEE Trans. Magn. 1991, 27, 4471-4475. [CrossRef]

19. Scholten, R. Enhanced laser shutter using a hard disk drive rotary voice-coil actuator. Rev. Sci. Instrum. 2007, 78, 026101. [CrossRef] [PubMed]

20. Yu, H.C.; Lee, T.Y.; Wang, S.J.; Lai, M.L.; Ju, J.J.; Huang, D.R.; Lin, S.K. Design of a voice coil motor used in the focusing system of a digital video camera. IEEE Trans. Magn. 2005, 41, 3979-3981.

21. Lotti, F.; Salmon, M.; Vassura, G.; Zucchelli, A. Selection of Linear Motors for High-Speed Packaging Machines. In Proceedings of the 2001 IEEE/ASME International Conference on Advanced Intelligent Mechatronics, Como, Italy, 8-12 July 2001; (Cat. No. 01TH8556). pp. 659-664.

22. Liu, Z.Z.; Luo, F.L.; Rahman, M.A. Robust and precision motion control system of linear-motor direct drive for high-speed XY table positioning mechanism. IEEE Trans. Ind. Electron. 2005, 52, 1357-1363. [CrossRef]

23. Wang, J.; Wang, W.; Atallah, K. A linear permanent-magnet motor for active vehicle suspension. IEEE Trans. Veh. Technol. 2010, 60, 55-63. [CrossRef]

24. Chladny, R.R.; Koch, C.R.; Lynch, A.F. Modeling automotive gas-exchange solenoid valve actuators. IEEE Trans. Magn. 2005, 41, 1155-1162. [CrossRef] 
25. Ebrahimi, B.; Khamesee, M.B.; Golnaraghi, F. Design of a Hybrid Electromagnetic/Hydraulic Damper for Automotive Suspension Systems. In Proceedings of the 2009 International Conference on Mechatronics and Automation, Malaga, Spain, 14-17 April 2009; pp. 3196-3200.

26. Dario, P.; Hannaford, B.; Menciassi, A. Smart surgical tools and augmenting devices. IEEE Trans. Robot. Autom. 2003, 19, 782-792. [CrossRef]

27. Narayanswamy, R.; Mahajan, D.P.; Bavisetti, S. Unified Coil Solenoid Actuator for Aerospace Application. In Proceedings of the 2012 Electrical Systems for Aircraft, Railway and Ship Propulsion, Bologna, Italy, 16-18 October 2012; pp. 1-5.

28. Boyd, C. Bio-Medical Injector Apparatus. U.S. Patent 4613328, 23 September 1986.

29. Nordson_Corporation. PICO P $\mu$ lse Contact Dispense Valves. Available online: https://www.nordson.com/ en/divisions/efd/products (accessed on 2 November 2019).

30. Zhou, C.; Li, J.; Duan, J.; Deng, G. The principle and physical models of novel jetting dispenser with giant magnetostrictive and a magnifier. Sci. Rep. 2015, 5, 18294. [CrossRef]

31. Nordson_Corporation. Liquidyn P-Jet Series Jetting Valves. Available online: https://www.nordson.com/en/ divisions/efd/products/ (accessed on 2 November 2019).

32. Mitchell, J. Linear motion devices: What is a voice-coil actuator? Laser Focus World 2018, 54, 41-43.

33. BEI_Kimco. Moving Magnet Voice Coil Actuators Offer Better Heat Displacement. Available online: https://news.thomasnet.com/companystory/ (accessed on 2 November 2019).

34. Encica, L.; Makarovic, J.; Lomonova, E.A.; Vandenput, A.J. Space mapping optimization of a cylindrical voice coil actuator. IEEE Trans. Ind. Appl. 2006, 42, 1437-1444. [CrossRef]

35. Hiemstra, D.B.; Parmar, G.; Awtar, S. Performance tradeoffs posed by moving magnet actuators in flexure-based nanopositioning. IEEE/ASME Trans. Mechatron. 2012, 19, 201-212. [CrossRef]

36. Ward, J.R.; Dahlgren, D.A.; Stobbs, T.J. Latching Solenoid with Improved Pull Force. U.S. Patent 6392516, 21 May 2002.

37. Kim, J.; Chang, J. A new electromagnetic linear actuator for quick latching. IEEE Trans. Magn. 2007, 43, 1849-1852. [CrossRef]

38. Zhou, C.; Li, J.; Duan, J.a.; Deng, G. Control and jetting characteristics of an innovative jet valve with zoom mechanism and opening electromagnetic drive. IEEE/ASME Trans. Mechatron. 2015, 21, 1185-1188. [CrossRef]

39. Kawase, Y.; Ohdachi, Y. Dynamic analysis of automotive solenoid valve using finite element method. IEEE Trans. Magn. 1991, 27, 3939-3942. [CrossRef]

40. Linear Solenoid Actuator. Available online: https://www.electronics-tutorials.ws/io/io_6.html (accessed on 5 November 2019).

41. Bruckner, F.; Vogler, C.; Bergmair, B.; Huber, T.; Fuger, M.; Suess, D.; Feischl, M.; Fuehrer, T.; Page, M.; Praetorius, D. Combining micromagnetism and magnetostatic Maxwell equations for multiscale magnetic simulations. J. Magn. Magn. Mater. 2013, 343, 163-168. [CrossRef] [PubMed]

42. Chapter 22: Maxwell 2D Technical Notes. In Maxwell ANSYS Online Help; ANSYS, Inc.: Canonsburg, PA, USA, 2012.

43. Nguyen, Q.H.; Han, Y.M.; Choi, S.B.; Hong, S.M. Dynamic characteristics of a new jetting dispenser driven by piezostack actuator. IEEE Trans. Electron Pack 2008, 31, 248-259. [CrossRef]

44. Free BH Curves of Low Carbon Steel-SAE1020. Available online: https://magweb.us/free-bh-curves/ (accessed on 6 November 2019).

(C) 2020 by the authors. Licensee MDPI, Basel, Switzerland. This article is an open access article distributed under the terms and conditions of the Creative Commons Attribution (CC BY) license (http://creativecommons.org/licenses/by/4.0/). 